\title{
The efficacy and safety of intravesical gemcitabine vs Bacille Calmette-Guérin for adjuvant treatment of non-muscle invasive bladder cancer: a meta- analysis
}

This article was published in the following Dove Press journal:

OncoTargets and Therapy

\author{
Ziqi Ye ${ }^{1, *}$ \\ Jie Chen ${ }^{2, *}$ \\ Yun Hong' \\ Wenxiu Xin ${ }^{3}$ \\ Si Yang' \\ Yuefeng Rao' \\ 'Department of Pharmacy, \\ The First Affiliated Hospital, College \\ of Medicine, Zhejiang University, \\ Hangzhou, People's Republic of China; \\ ${ }^{2}$ The Second Affiliated Hospital, \\ College of Medicine, Zhejiang \\ University, Hangzhou, People's \\ Republic of China; ${ }^{3}$ Laboratory of \\ Clinical Pharmacy, Zhejiang Cancer \\ Hospital, Hangzhou, People's Republic \\ of China \\ *These authors contributed equally \\ to this work
}

\begin{abstract}
Objective: Several studies have compared the safety and efficacy of intravesical gemcitabine (Gem) with Bacille Calmette-Guérin (BCG) for non-muscle invasive bladder cancer. However, the results are not consistent. We carried out a meta-analysis to provide a more comprehensive analysis of the efficacy and safety of these 2 drugs.

Methods: We searched PubMed, EMBASE, Cochrane Library, Clinical Trials.gov, and reference lists. Randomized controlled trials and retrospective controlled trials comparing intravesical Gem and BCG in adjuvant therapy for non-muscle invasive bladder cancer published in English were included in this study. The strength of association was weighed by pooled risk ratio (RR) with $95 \%$ CIs. Sensitivity analysis was performed to examine whether the findings of the meta-analysis were robust.

Results: We analyzed 386 subjects from 5 pooled trials. Compared with BCG, intravesical Gem had lower incidence of dysuria (overall $\mathrm{RR}=0.31,95 \% \mathrm{CI}: 0.16,0.61, I^{2}=0 \%, p=0.001$ ) and hematuria (overall RR $=0.27,95 \% \mathrm{CI}: 0.11,0.71, I^{2}=0 \%, p=0.008$ ). There were no statistical differences in risk of recurrence, progression, incidence of fever, and any adverse events between intravesical Gem and BCG therapy ( $p>0.05$ ). No publication bias was found.

Conclusion: This meta-analysis suggests that intravesical Gem may have similar efficacy and lower incidence of dysuria and hematuria compared with BCG. Nevertheless, we recommend additional high-quality randomized controlled trials to confirm these results.
\end{abstract}

Keywords: intravesical therapy, non-muscle invasive bladder cancer, NMIBC, gemcitabine, Bacille Calmette-Guérin, meta-analysis

\section{Introduction}

Globally, 390,000 new cases and 150,000 deaths due to bladder cancer are reported each year. ${ }^{1}$ In 2017, there were 79,030 new cases in the USA. ${ }^{2}$ Among middle-aged and older men, bladder cancer is the second most common type of cancer after prostate cancer.

In developed areas of the world, such as Western Europe and North America, bladder cancers are predominantly urothelial. About $70 \%$ of new urothelial bladder cancer cases are classified as NMIBC, including Ta, T1, and Tis tumors, which account for $\sim 70 \%, 20 \%$, and $10 \%$ of NMIBCs, respectively. ${ }^{3}$ These tumors are usually diagnosed by cystoscopic visualization and treated by transurethral resection of bladder tumor (TURBT). An estimated $40 \%-80 \%$ of NMIBC recur within $6-12$ months when managed with TURBT without additional therapy, and $10 \%-25 \%$ of the patients
Correspondence: Yuefeng Rao The First Affiliated Hospital, College of Medicine, Zhejiang University, 79 Qingchun Road, Hangzhou 310003, People's Republic of China

Tel +86 57I 87236675

Fax +86 57I 87236675

Email raoyf@zju.edu.cn 
progress to muscle invasive, regional, or metastatic disease. ${ }^{4}$ Intravesical therapy enables delivery of high local concentrations of a therapeutic agent within the bladder, which could potentially destroy viable tumor cells that remain following TURBT. Intravesical therapy is generally used as adjuvant therapy following TURBT.

BCG, an attenuated form of Mycobacterium bovis, is the most commonly used agent for intravesical therapy. Gemcitabine (Gem) is a relatively new anticancer drug with activity against metastatic bladder cancer. Intravesical Gem is efficacious and potentially safe for NMIBC patients as it offers complete tumor remission in $23 \%-56 \%$ of patients and 1 -year recurrence-free survival in up to $21 \%$ of patients..$^{5-8}$

In 2012, a systematic review indicated that Gem had similar effects to BCG in NMIBC patients, but its safety profile was not reported. ${ }^{9}$ Only 3 randomized controlled trials (RCTs) were included in the review and no meta-analysis was performed. Moreover, the results from studies comparing the efficacy and safety of intravesical Gem with BCG in NMIBC patients have not yielded a consistent conclusion. Some studies found significant differences between the 2 treatments, while others did not find any significant difference. Therefore, we carefully designed a meta-analysis to clarify the differences, in terms of efficacy and safety, between Gem and $\mathrm{BCG}$ in NMIBC patients.

\section{Methods}

\section{Search strategy}

To search for all the studies that compared intravesical Gem and BCG, we conducted a comprehensive literature search on PubMed, EMBASE, the Cochrane Library, and Clinical Trials.gov (http://ClinicalTrials.gov/) for reported clinical trials published up to April, 2018. The search terms included: "Gemcitabine," "BCG," "Bacille Calmette-Guérin," "bladder cancer," "bladder carcinoma," "bladder tumor." All studies performed in humans were included. We also screened all the reference lists of the retrieved articles. We also retrieved additional studies by handsearching in relevant journals. Only studies published in English were included.

\section{Inclusion criteria and exclusion criteria}

Studies were selected according to the Preferred Reporting Items for Systematic Reviews and Meta-Analyses statement. ${ }^{10}$ Clinical trials that met the following criteria were included:

1. Randomized Phase II, III, and IV trials

2. Adults with NMIBC (Tis, Ta, or T1) who underwent TURBT and received intravesical therapy for the treatment of bladder cancer
3. Participants who received intravesical Gem compared to $\mathrm{BCG}$

4. Events and event rates and sample sizes available for drug efficacy and safety.

Exclusion criteria were as follows: 1) reviews, 2) animal research, 3) studies with overlapping data, and 4) studies without odds ratio (OR), risk ratio (RR), or hazard ratio (HR) with 95\% CI.

\section{Data extraction and quality assessment}

The literature screening, data extraction, and quality assessment of the trials were independently conducted by 2 reviewers. If reviewers disagreed, a third reviewer intervened until a consensus was reached. The following information was extracted from each article: first author's name, year of publication, study type, journal, underlying disease, trial phase, number of enrolled patients, treatment and control cohort, the number of patients with recurrence, progression, and events of adverse effects. The quality of the methodology in each trial was assessed according to the Jadad criteria. ${ }^{11}$ The quality of each included trial was graded, with high-quality trials classified as those with a score $\geq 3$ and low-quality trials classified as those with a score of $\leq 2$. The quality of the methodology in retrospective studies was assessed by the Newcastle-Ottawa Scale criteria (http://www. ohri.ca/programs/clinical epidemiology/oxford.asp) (range 0-9 stars). The quality of each trial included was graded, with high-quality trials classified as those with a score $\geq 7$ stars.

\section{Statistical analysis}

Data on patients with recurrence, progression, and events of adverse effects were extracted from all of the included trials, and the RR and 95\% CIs were calculated to assess the strength of the association of these 2 drugs with risk of recurrence, progression, and adverse events. The heterogeneity test was assessed by the $Q$ statistic and $I^{2}$ statistic. $I^{2}>40 \%$ indicated statistically significant heterogeneity and that the statistical method should be changed to random-effect model. In other cases, a fixed-effect model was used. Analysis of subgroups was carried out according to clinical characteristics. Sensitivity analysis was performed to examine whether the findings in the meta-analysis were robust. Publication bias was investigated by a Begg modified funnel plot. An asymmetric plot suggested possible publication bias. Funnel-plot asymmetricity was assessed by the method of Begg adjusted rank correlation test ${ }^{12}$ and Egger regression test. ${ }^{13}$ A statistical test with a $p$-value $<0.05$ was considered significant. All statistical analyses were performed by using STATA statistical version 12.0 (Stata Corporation, College Station, TX, USA). All $p$-values were 2-sided. 


\section{Results}

\section{Characteristics of studies included in this study}

Our search yielded a total of 545 potentially relevant clinical trials of bladder cancer with intravesical Gem therapy. After reviewing and screening, 5 primary studies, which included 386 subjects, met our inclusion criteria ${ }^{14-18}$ and were pooled for the meta-analysis (Figure S1). The baseline characteristics of each trial are shown in Table 1; 3 RCTs and 1 retrospective controlled trial were included. All trials included were open label. The quality of the included RCTs (Jadad scores) ranged from 1 to 5, while the score of the 1 retrospective controlled trial was of high-quality with Newcastle-Ottawa Scale score of 8 . According to the eligibility criteria of the majority of the trials, patients with impaired renal, hepatic, or bone marrow function were excluded and most of the patients had Eastern Cooperative Oncology Group performance status scores of 0 or 1 (on a scale from 0 to 5, with higher scores indicating greater disability). The guidelines of the Preferred Reporting Items for Systematic Reviews and Meta-Analyses statement were followed in this meta-analysis.

\section{Recurrence risk of intravesical Gem and BCG therapy}

A total of 283 subjects who were treated with Gem or BCG in 4 trials were used for the analysis of recurrence risk. As shown in Figure 1, the overall recurrence RR between Gem and BCG therapy was 1.17 (95\% CI: $0.83,1.67, I^{2}=10.3 \%$ ). The results showed no statistical difference in recurrence risk between intravesical Gem and BCG ( $p=0.367)$.

\section{Progression risk of intravesical Gem and BCG therapy}

A total of 283 subjects that were treated with Gem or BCG in 4 trials were used for the analysis of progression risk, which is an important indicator of curative effects. As shown in Figure 2, the overall progression RR between Gem and BCG therapy was 1.02 ( $95 \%$ CI: $\left.0.41,2.56, I^{2}=0 \%\right)$. The results showed no statistical difference in the progression risk between intravesical Gem and BCG ( $p=0.961)$. Data from the Porena et al' $\mathrm{s}^{14}$ trial was excluded automatically by STATA software, because the progression rate in both Gem and $\mathrm{BCG}$ groups was $0 \%$.

\section{Adverse effects of intravesical Gem and BCG therapy}

A total of 386 subjects who were treated with Gem or BCG from 5 trials were used for the analysis of adverse effects (dysuria, fever, hematuria, and any adverse events). As shown in Figure 3, the RR for overall dysuria, fever, hematuria, and any adverse events between Gem and BCG therapy were 0.31 (95\% CI: $\left.0.16,0.61, I^{2}=0 \%\right), 0.42$ (95\% CI: $\left.0.12,1.39, I^{2}=0 \%\right), 0.27$ (95\% CI: $0.11,0.71, I^{2}=0 \%$ ), and 0.55 (95\% CI: $\left.0.25,1.20, I^{2}=63.9 \%\right)$, respectively. Patients receiving intravesical Gem therapy had significantly lower incidence of dysuria and hematuria compared to those receiving intravesical BCG treatment ( $p=0.001$ and 0.008 , respectively). However, there was no statistical difference in risk of fever and any adverse events between intravesical Gem and BCG ( $p=0.153$ and 0.134 , respectively). Although the result of any adverse events showed heterogeneity $\left(I^{2}=63.9 \%\right.$, $\left.p_{\text {heterogeneity }}=0.040\right)$, sensitivity analysis confirmed that this conclusion was robust (Figure S2).

\section{Sensitivity analysis}

As shown in Figure S2, the influential analysis showed that no particular study affected the overall significance of the pooled estimates and the findings in the meta-analysis were robust.

\section{Publication bias}

As shown in Figure S3, the shape of the funnel plot did not display any evidence of apparent asymmetry. Furthermore,

Table I Characteristics of all studies

\begin{tabular}{|c|c|c|c|c|c|c|c|c|c|c|}
\hline \multirow[t]{2}{*}{ Study } & \multirow[t]{2}{*}{ Country } & \multirow[t]{2}{*}{ Stage } & \multirow[t]{2}{*}{$\begin{array}{l}\text { Number, } \\
\text { Gem/BCG }\end{array}$} & \multirow[t]{2}{*}{$\begin{array}{l}\text { Age (year), } \\
\text { Gem/BCG }\end{array}$} & \multicolumn{2}{|c|}{$\begin{array}{l}\text { Interventions } \\
\text { (weekly) }\end{array}$} & \multirow[t]{2}{*}{ Outcomes } & \multirow[t]{2}{*}{$\begin{array}{l}\text { Follow-up } \\
\text { (years) }\end{array}$} & \multirow[t]{2}{*}{$\begin{array}{l}\text { Jadad } \\
\text { r score }\end{array}$} & \multirow[t]{2}{*}{$\begin{array}{l}\text { NOS } \\
\text { score }\end{array}$} \\
\hline & & & & & $\begin{array}{l}\text { Gem } \\
\text { (mg) }\end{array}$ & BCG & & & & \\
\hline Bendary et al, ${ }^{17} 2011$ & Egypt & $\mathrm{Ta}-\mathrm{TI}$ & $40 / 40$ & 56.2 & 2,000 & $6 \times 10^{8} \mathrm{CFU}$ & rr; PR; AE & 2 & 1 & \\
\hline Porena et al, ${ }^{14} 2010$ & Italy & $\mathrm{Ta}-\mathrm{TI}$ & $32 / 32$ & $70.2 / 68.7$ & 2,000 & $5 \times 10^{8} \mathrm{CFU}$ & rr; PR; AE & 2 & 5 & \\
\hline Gacci et al, ${ }^{15} 2006$ & Italy & TI & $9 / 10$ & $74 / 73.6$ & 2,000 & $5 \times 10^{8} \mathrm{CFU}$ & rr; PR; AE & 2 & 1 & \\
\hline Gontero et al, ${ }^{16} 2013$ & $\begin{array}{l}\text { Italy, Germany, } \\
\text { USA }\end{array}$ & $\mathrm{Ta}-\mathrm{TI}$ & $61 / 59$ & $67.4 / 67.5$ & 2,000 & $2.2-6.4 \times 10^{8} \mathrm{CFU}$ & rr; PR; AE & 4 & 3 & \\
\hline Prasanna et al, ${ }^{18} 2017$ & Australia & $\mathrm{Ta}-\mathrm{TI}$ & $51 / 52$ & $78 / 77$ & 2,000 & $5 \times 10^{8} \mathrm{CFU}$ & DFS; AE & 1.25 & & 8 stars \\
\hline
\end{tabular}

Abbreviations: AE, adverse event; BCG, Bacille Calmette-Guérin; CFU, colony forming unit; DFS, disease-free survival; Gem, gemcitabine; PR, progression rate; rr, recurrence rate. 


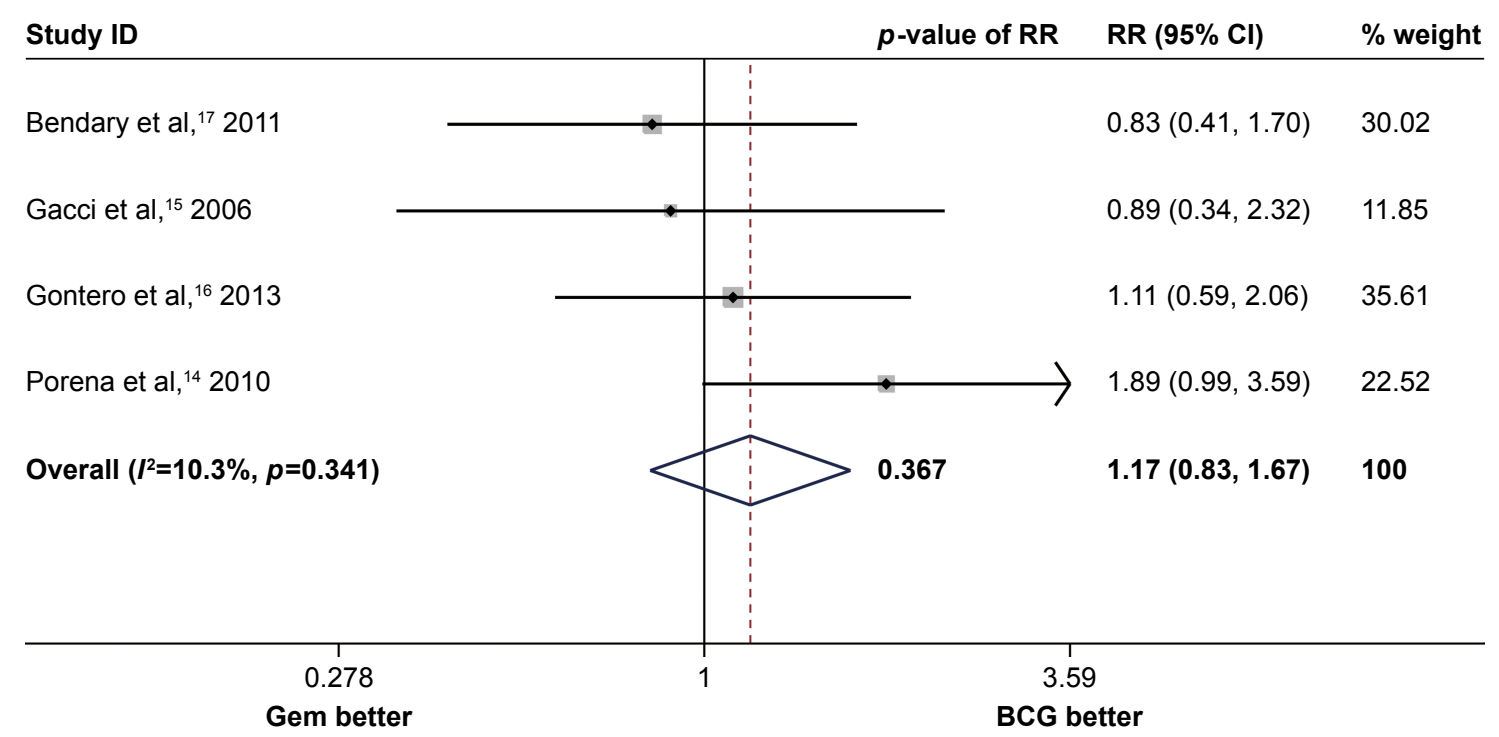

Figure I Annotated forest plot for meta-analysis of recurrence risk of intravesical Gem and BCG therapy.

Notes: Summary of recurrence RR between intravesical Gem and BCG therapy were calculated with fixed-effect model. Size of squares is directly proportional to amount of information available.

Abbreviations: BCG, Bacille Calmette-Guérin; Gem, gemcitabine; RR, risk ratio.

the formal tests showed no evidence of significant publication bias ( $p=0.734$ for the Begg's test; $p=0.508$ for the Egger's test).

\section{Discussion}

BCG is the most commonly used agent for adjuvant intravesical therapy in NMIBC patients after TURBT. A number of other intravesical agents have been compared with BCG; most were inferior and none were found to be superior. ${ }^{19-23}$
Three relatively small randomized trials were pooled to compare the efficacy of Gem with BCG, but the safety aspect was not mentioned and no meta-analysis was performed. ${ }^{24}$ Until now, the results of these trials are not compelling enough to support any definitive conclusions about the usage of Gem. Here, we performed a meta-analysis on the efficacy and safety of intravesical Gem and BCG in NMIBC patients based on 5 clinical trials which included a total 386 bladder cancer patients. Our results indicated that 1) there was no statistical

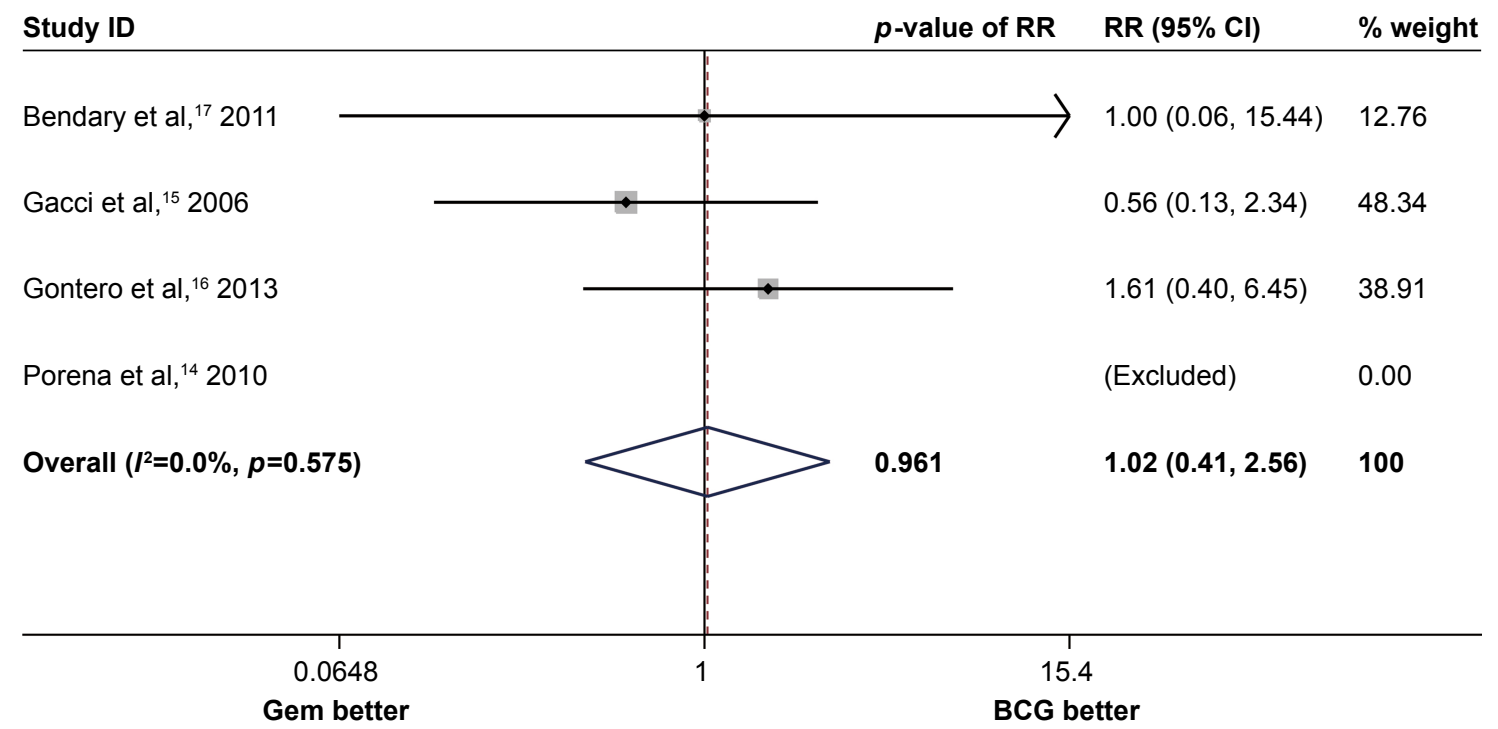

Figure 2 Annotated forest plot for meta-analysis of progression risk of intravesical Gem and BCG therapy.

Notes: Summary of progression RR between intravesical Gem and BCG therapy were calculated with fixed-effect model. Size of squares is directly proportional to amount of information available.

Abbreviations: BCG, Bacille Calmette-Guérin; Gem, gemcitabine; RR, risk ratio. 


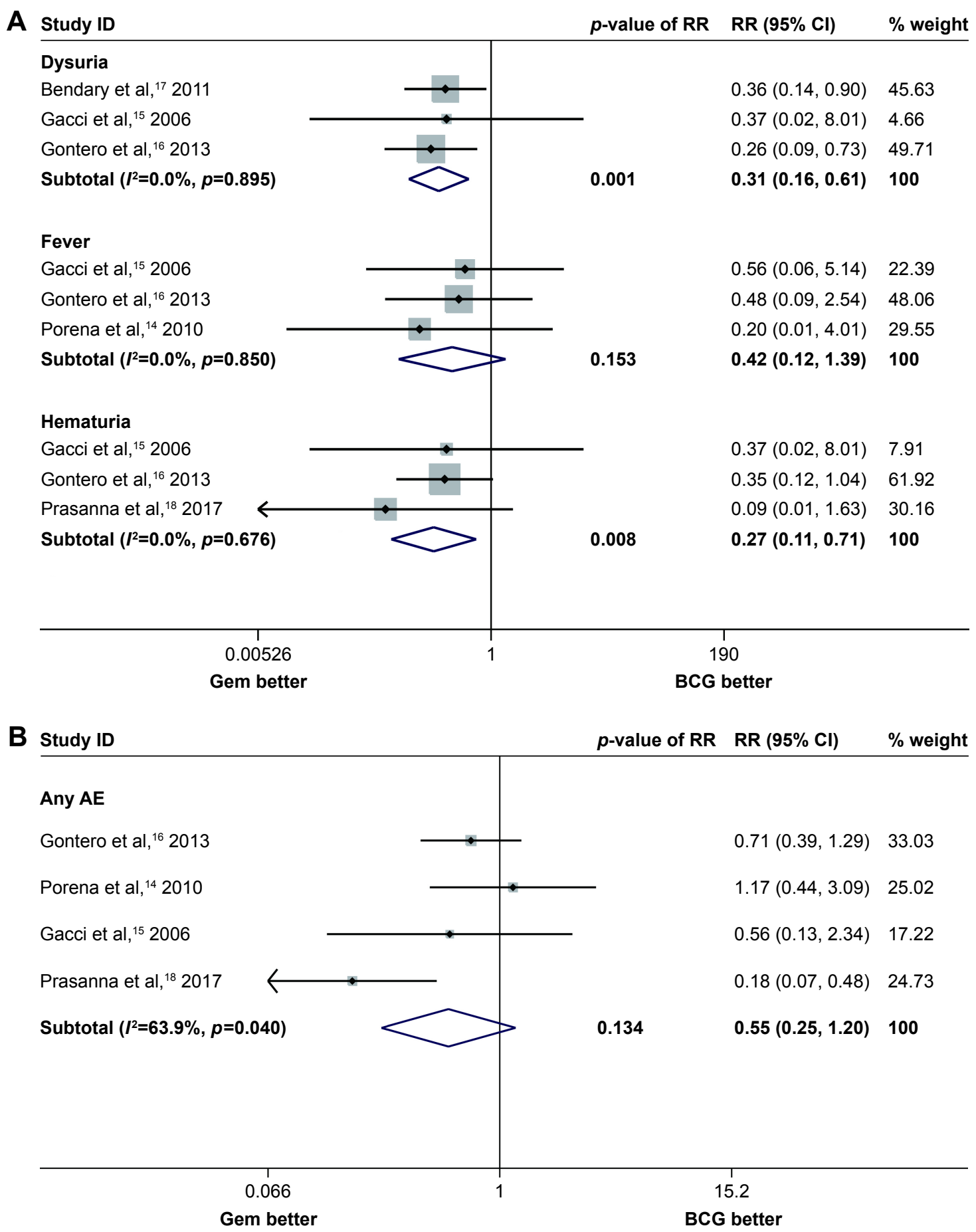

Figure 3 Annotated forest plot for meta-analysis of the risk of adverse effects for intravesical Gem and BCG therapy.

Notes: Summary RR for adverse effects between intravesical Gem and BCG therapy (A dysuria, fever, hematuria, and B any adverse events) were calculated with fixed-effect model or random-effect model. Size of squares was directly proportional to amount of information available. Weights are from random-effects analysis.

Abbreviations: BCG, Bacille Calmette-Guérin; Gem, gemcitabine; RR, risk ratio.

difference in recurrence risk between intravesical Gem and BCG; 2) there was no statistical difference in progression risk between intravesical Gem and BCG; and 3) intravesical Gem therapy had significantly lower incidence of dysuria and hematuria compared to BCG, but there was no statistical difference in risk of fever and any adverse events. However, these conclusions should be interpreted with caution and need to be verified in future studies.

When bladder cancer is confined to the lining of the bladder, surgical removal of the tumor is the first treatment 
choice. However the tumors may recur, hence intravesical therapy in which agents are instilled directly into the bladder to prevent tumor recurrence is often used following surgery. BCG is a classical intravesical agent used to prevent recurrence by stimulating the body's immune system to kill any residual cancer cells ${ }^{25-27}$ or by acting directly on the tumor cells. $^{28} \mathrm{~A}$ relatively new drug in this disease is Gem. In a 2007 meta-analysis, a single-dose postoperative instillation of chemotherapy provided an average absolute benefit of $17 \%$ reduction in early tumor recurrence..$^{29}$ Until now, there is no consensus on which drug may offer results that are superior to BCG. Therefore, intravesical BCG remains the standard regimen for NMIBC patients. However, as was shown in this meta-analysis, intravesical Gem might have similar efficacy to BCG. In addition to efficacy, clinicians also pay attention to adverse effects. This meta-analysis showed that intravesical Gem might have lower incidence of dysuria and hematuria, indicating that it may offer good side effect profile for clinical use. However, only few studies were included in this meta-analysis, and therefore more highquality RCTs are required to confirm these results.

The results of our study are in general agreement with those performed by Shelley et al..$^{9}$ In the systematic review, it was found that intravesical Gem had similar efficacy to BCG. But a meta-analysis was not performed and the safety between the 2 drugs was not compared. Our study compared the safety of these 2 drugs, and a meta-analysis was performed to draw more comprehensive results. Moreover, our study included more relevant articles; although the total number of studies included was still small, the findings from this analysis might stimulate further investigations.

It is known that the risk of recurrence and prognosis are different between Ta and T1 NMIBC, and the ratio of Ta and T1 may affect the recurrence risk. In line with results from the trial of Bendary et al ${ }^{17}$ included in this metaanalysis, the Ta group had a recurrence rate of $26.31 \%$ in BCG group and $22.22 \%$ in Gem group. The T1 group had higher recurrence rate of $33.33 \%$ in $\mathrm{BCG}$ group and $27.27 \%$ in Gem group. ${ }^{17}$ Although the ratio of Ta and T1 in the trials included was different, this study used the overall recurrence risk since there was no statistically significant difference between Gem and BCG group in each trial. Since we currently lack relevant RCTs to compare recurrence risk of BCG and Gem in either Ta or T1 group separately, we could not draw any conclusion about which drug has a lower recurrence risk in Ta or T1 group. All intravesical therapies may cause bladder irritation (such as dysuria and hematuria). In addition, systemic effects may occur if the agent is absorbed through the mucosa. In a systematic review, toxicities associated with intravesical BCG included increased urinary frequency $(71 \%)$, cystitis $(67 \%)$, fever (25\%), and hematuria $(23 \%) \cdot{ }^{30}$ In this meta-analysis, the results showed that Gem had a significantly lower incidence of dysuria and hematuria. Although these results should be interpreted with caution, they suggest that Gem might be a promising agent for NMIBC patients.

Despite the proven efficacy of intravesical therapy, some patients experience tumor recurrence. In such cases, cystectomy is considered for treatment, or additional intravesical therapy is used for patients who refuse cystectomy or are not medically fit for cystectomy. In recent years, several studies on combination therapy of Gem and other chemical agents for intravesical therapy in patients with NMIBC have be published, such as mitomycin. ${ }^{31-33}$ Usage of many drugs with different mechanisms increases the chances of killing tumor cells and decreases the chances of cell resistance to therapy. Although the combination of intravesical Gem and mitomycin might increase the incidence of adverse events, it may offer long-term recurrence-free survival to some patients with recurrent NMIBC who refuse cystectomy or are not fit for cystectomy. Consequently, more high-quality RCTs to confirm the effectiveness of combination intravesical therapy for NMIBC patients are advocated.

Publication bias may introduce false-positive results in a meta-analysis. Therefore, to avoid possible bias, all studies included were carefully assessed. Begg's and Egger's tests for detecting publication bias were performed, and no evidence of bias was found. Sensitivity analysis was performed to examine whether the findings of the meta-analysis were robust. The results showed that no particular study affected the overall significance of the pooled estimates and that the findings in this meta-analysis were robust. The results of sensitivity and publication bias analyses indicate that the conclusions of our study are credible.

The present meta-analysis should be interpreted with caution, and several limitations merit consideration. First, the number of clinical trials was small, especially due to the lack of high-quality RCTs to compare the recurrence risk of Gem and BCG in Ta or T1 group separately. Second, although the dosage and schedule of the 2 drugs in all 5 controlled trials included in this review were consistently used, more rigorously designed experiments are required. Third, the 5 clinical trials used in this analysis were not double-blinded, which may affect the outcomes. Fourth, publication bias cannot be entirely excluded, hence similar to other meta-analyses, publication bias might have the potential to distort the conclusion since all the included studies in this meta-analysis were in English and other small-sized studies were included. 


\section{Conclusion}

This meta-analysis indicates that intravesical Gem may have similar efficacy and lower incidence of dysuria and hematuria compared with BCG. More large-scale studies incorporating various covariates should be performed to further elucidate the efficacy and safety of Gem compared with BCG.

\section{Acknowledgment}

This work was supported by the Natural Science Foundation of Zhejiang Province, China (Grant number LYY18H310002), Department of Health of Zhejiang Province, China (No 2015RCB006) and the Natural Science Foundation of Zhejiang Province, China (Grant number LY17H310002).

\section{Disclosure}

The authors report no conflicts of interest in this work.

\section{References}

1. Antoni S, Ferlay J, Soerjomataram I, Znaor A, Jemal A, Bray F. Bladder cancer incidence and mortality: a global overview and recent trends. Eur Urol. 2017;71(1):96-108.

2. Siegel RL, Miller KD, Jemal A. Cancer statistics, 2017. CA Cancer J Clin. 2017;67(1):7-30.

3. Kirkali Z, Chan T, Manoharan M, et al. Bladder cancer: epidemiology, staging and grading, and diagnosis. Urology. 2005;66(6 Suppl 1): 4-34.

4. Soukup V, Capoun O, Cohen D, et al. Prognostic performance and reproducibility of the 1973 and 2004/2016 World Health Organization grading classification systems in non-muscle-invasive bladder cancer: a European Association of Urology non-muscle invasive bladder cancer guidelines panel systematic review. Eur Urol. Apr 272017.

5. Gontero P, Casetta G, Maso G, et al. Phase II study to investigate the ablative efficacy of intravesical administration of gemcitabine in intermediate-risk superficial bladder cancer (SBC). Eur Urol. 2004; 46(3):339-343.

6. Dalbagni G, Russo P, Bochner B, et al. Phase II trial of intravesical gemcitabine in bacille Calmette-Guerin-refractory transitional cell carcinoma of the bladder. J Clin Oncol. 2006;24(18):2729-2734.

7. Serretta V, Galuffo A, Pavone C, Allegro R, Pavone-MacAluso M. Gemcitabine in intravesical treatment of $\mathrm{Ta}-\mathrm{T} 1$ transitional cell carcinoma of bladder: phase I-II study on marker lesions. Urology. 2005; 65(1):65-69.

8. Bartoletti R, Cai T, Gacci M, et al. Intravesical gemcitabine therapy for superficial transitional cell carcinoma: results of a phase II prospective multicenter study. Urology. 2005;66(4):726-731.

9. Shelley MD, Jones G, Cleves A, Wilt TJ, Mason MD, Kynaston HG. Intravesical gemcitabine therapy for non-muscle invasive bladder cancer (NMIBC): a systematic review. BJU Int. 2012;109(4):496-505.

10. Moher D, Liberati A, Tetzlaff J, Altman DG, Group P. Preferred reporting items for systematic reviews and meta-analyses: the PRISMA statement. BMJ. 2009;339:b2535.

11. Jadad AR, Moore RA, Carroll D, et al. Assessing the quality of reports of randomized clinical trials: is blinding necessary? Control Clin Trials. 1996;17(1):1-12.

12. Begg CB, Mazumdar M. Operating characteristics of a rank correlation test for publication bias. Biometrics. 1994;50(4):1088-1101.

13. Egger M, Davey Smith G, Schneider M, Minder C. Bias in meta-analysis detected by a simple, graphical test. BMJ. 1997;315(7109):629-634.

14. Porena M, Del Zingaro M, Lazzeri M, et al. Bacillus Calmette-Guerin versus gemcitabine for intravesical therapy in high-risk superficial bladder cancer: a randomised prospective study. Urol Int. 2010;84(1): $23-27$.
15. Gacci M, Bartoletti R, Cai T, et al. Intravesical gemcitabine in BCGrefractory T1G3 transitional cell carcinoma of the bladder: a pilot study. Urol Int. 2006;76(2):106-111.

16. Gontero P, Oderda M, Mehnert A, et al. The impact of intravesical gemcitabine and 1/3 dose Bacillus Calmette-Guerin instillation therapy on the quality of life in patients with nonmuscle invasive bladder cancer: results of a prospective, randomized, phase II trial. J Urol. 2013;190(3):857-862.

17. Bendary L, Khalil S, Shahin A, Nawar N. 1655 Intravesical gemcitabine versus Bacillus Calmette-Guerin (BCG) in treatment of non-muscle invasive bladder cancer: short term comparative study. J Urol. 2011; 185(4):e664-e665.

18. Prasanna T, Craft P, Balasingam G, Haxhimolla H, Pranavan G. Intravesical gemcitabine versus intravesical Bacillus Calmette-Guérin for the treatment of non-muscle invasive bladder cancer: an evaluation of efficacy and toxicity. Front Oncol. 2017;7:260.

19. Sylvester RJ, van der Meijden AP, Witjes JA, Kurth K. Bacillus calmetteguerin versus chemotherapy for the intravesical treatment of patients with carcinoma in situ of the bladder: a meta-analysis of the published results of randomized clinical trials. J Urol. 2005; 174(1):86-91; discussion 91-82.

20. Martinez-Pineiro JA, Jimenez Leon J, Martinez-Pineiro L Jr, et al. Bacillus Calmette-Guerin versus doxorubicin versus thiotepa: a randomized prospective study in 202 patients with superficial bladder cancer. J Urol. 1990;143(3):502-506.

21. Shelley MD, Court JB, Kynaston H, Wilt TJ, Coles B, Mason M. Intravesical bacillus Calmette-Guerin versus mitomycin $\mathrm{C}$ for $\mathrm{Ta}$ and T1 bladder cancer. Cochrane Database Syst Rev. 2003;3:CD003231.

22. Bohle A, Bock PR. Intravesical bacille Calmette-Guerin versus mitomycin $\mathrm{C}$ in superficial bladder cancer: formal meta-analysis of comparative studies on tumor progression. Urology. 2004;63(4):682-686; discussion 686-687.

23. de Reijke TM, Kurth KH, Sylvester RJ, et al. Bacillus Calmette-Guerin versus epirubicin for primary, secondary or concurrent carcinoma in situ of the bladder: results of a European Organization for the Research and Treatment of Cancer - Genito-Urinary Group Phase III Trial (30906). J Urol. 2005;173(2):405-409.

24. Jones G, Cleves A, Wilt TJ, Mason M, Kynaston HG, Shelley M. Intravesical gemcitabine for non-muscle invasive bladder cancer. Cochrane Database Syst Rev. 2012;1:CD009294.

25. Prescott S, Jackson AM, Hawkyard SJ, Alexandroff AB, James K. Mechanisms of action of intravesical bacille Calmette-Guerin: local immune mechanisms. Clin Infect Dis. 2000;31(Suppl 3):S91-S93.

26. Bohle A, Brandau S. Immune mechanisms in bacillus CalmetteGuerin immunotherapy for superficial bladder cancer. J Urol. 2003; 170(3):964-969.

27. Mungan NA, Witjes JA. Bacille Calmette-Guerin in superficial transitional cell carcinoma. Br J Urol. 1998;82(2):213-223.

28. Jackson A, Alexandroff A, Fleming D, Prescott S, Chisholm G, James K. Bacillus-calmette-guerin (bcg) organisms directly alter the growth of bladder-tumor cells. Int J Oncol. 1994;5(3):697-703.

29. Hall MC, Chang SS, Dalbagni G, et al. Guideline for the management of nonmuscle invasive bladder cancer (stages Ta, T1, and Tis): 2007 update. J Urol. 2007;178(6):2314-2330.

30. Shelley MD, Court JB, Kynaston H, Wilt TJ, Fish RG, Mason M. Intravesical Bacillus Calmette-Guerin in Ta and T1 bladder cancer. Cochrane Database Syst Rev. 2000;4:CD001986.

31. Maymi JL, Saltsgaver N, O’Donnell MA. Intravesical sequential gemcitabine-mitomycin chemotherapy as salvage treatment for patients with refractory superficial bladder cancer. J Urol. 2006; 175(Suppl 4):271.

32. Lightfoot AJ, Breyer BN, Rosevear HM, Erickson BA, Konety BR, O’Donnell MA. Multi-institutional analysis of sequential intravesical gemcitabine and mitomycin $\mathrm{C}$ chemotherapy for non-muscle invasive bladder cancer. Urol Oncol. 2014;32(1):35.e15-e39.

33. Cockerill PA, Knoedler JJ, Frank I, Tarrell R, Karnes RJ. Intravesical gemcitabine in combination with mitomycin $\mathrm{C}$ as salvage treatment in recurrent non-muscle invasive bladder cancer. BJU Int. 2016;117(3): 456-462. 


\section{Supplementary materials}

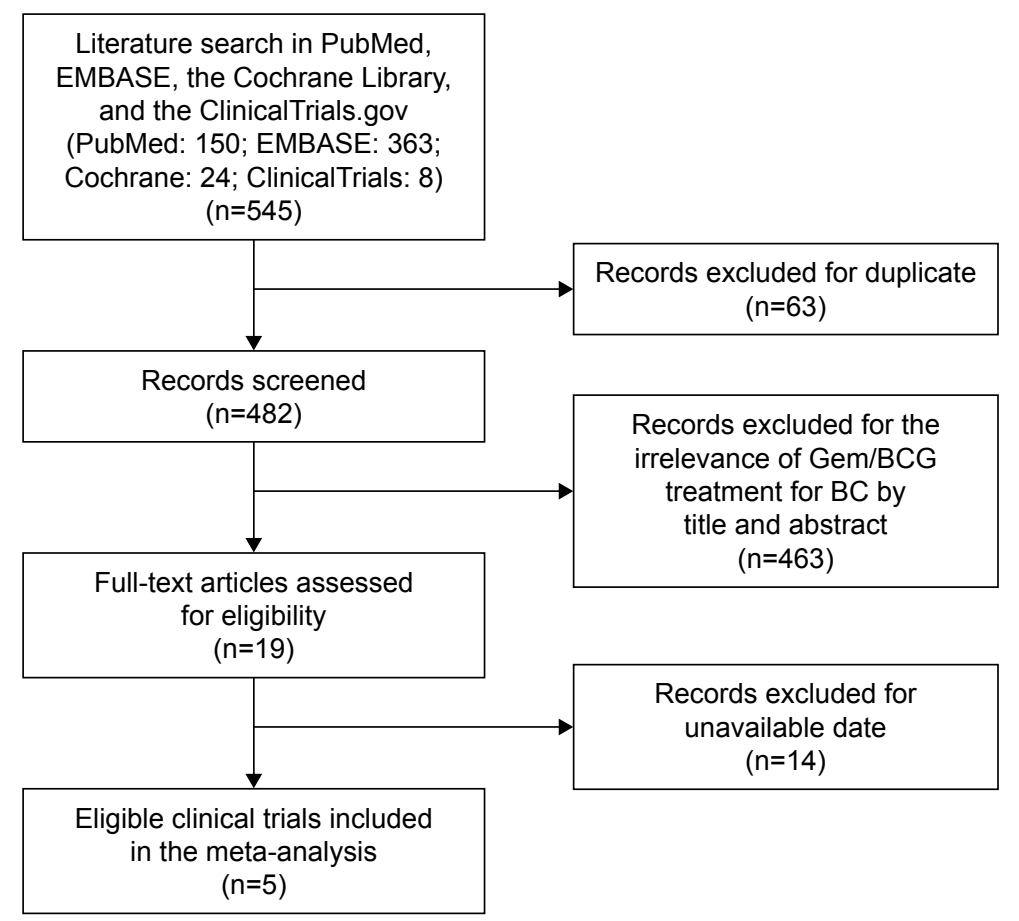

Figure SI Flow chart for eligible studies.

Abbreviations: RCTs, randomized controlled trials; Gem, gemcitabine; BCG, Bacille Calmette-Guérin; BC, bladder cancer.

Meta-analysis estimates, given named study is omitted

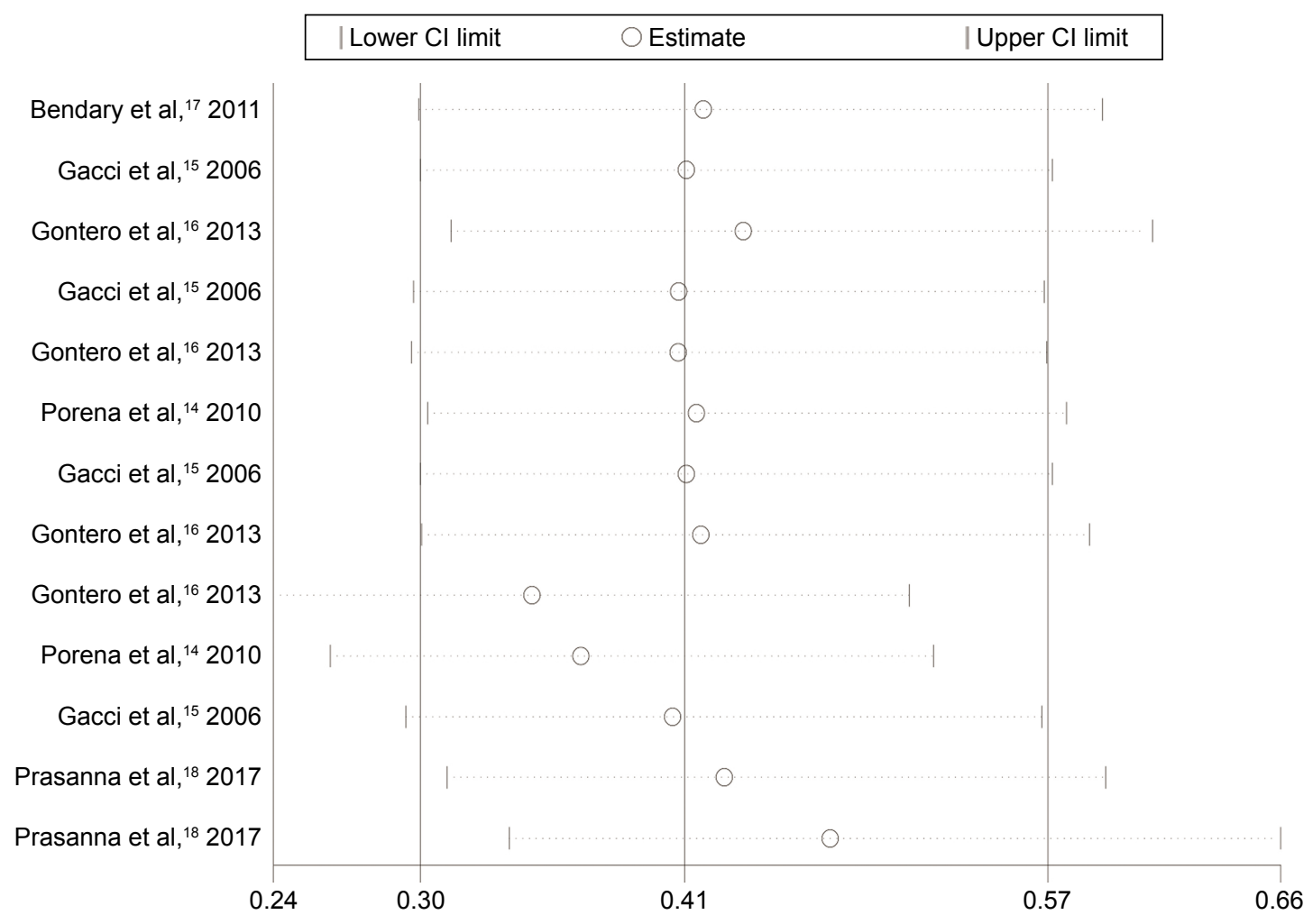

Figure S2 Sensitivity analysis of all clinical trials included. 


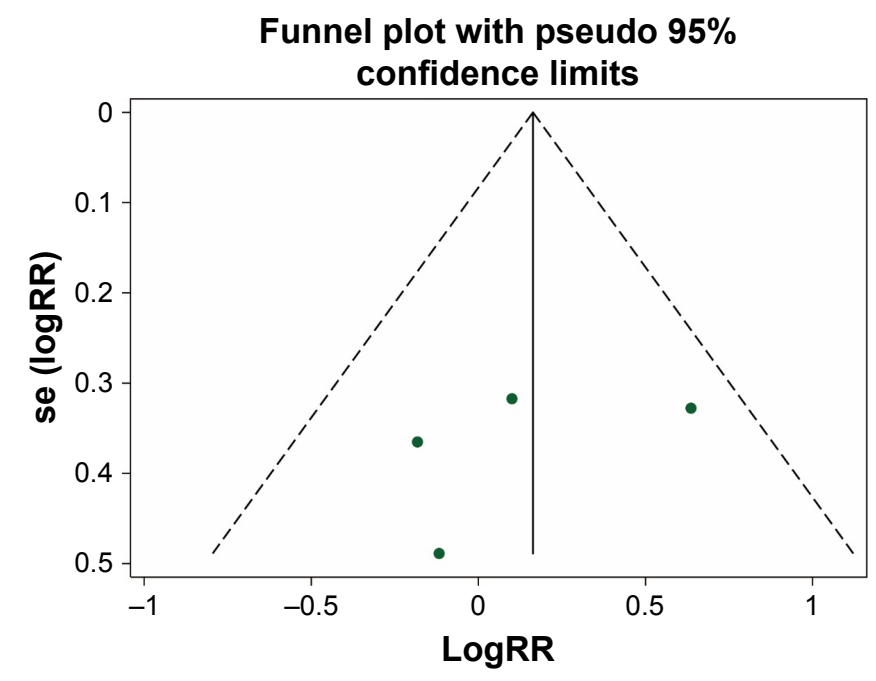

Figure S3 Publication bias risk.

Abbreviations: RR, risk ratio; se, standard error of the mean.

\section{Publish your work in this journal}

OncoTargets and Therapy is an international, peer-reviewed, open access journal focusing on the pathological basis of all cancers, potential targets for therapy and treatment protocols employed to improve the management of cancer patients. The journal also focuses on the impact of management programs and new therapeutic agents and protocols on
Dovepress

patient perspectives such as quality of life, adherence and satisfaction. The manuscript management system is completely online and includes a very quick and fair peer-review system, which is all easy to use. Visit http://www.dovepress.com/testimonials.php to read real quotes from published authors.

\footnotetext{
Submit your manuscript here: http://www.dovepress.com/oncotargets-and-therapy-journal
} 